\title{
Oral herbal supplement containing magnesium sulfate improve metabolic control and insulin resistance in non-diabetic overweight patients: A randomized double blind clinical trial
}

\author{
Mehrdad Solati ${ }^{1}$, Leila Kazemi ${ }^{1}$, Naghi Shahabi Majd ${ }^{2}$, Mansoor Keshavarz $^{3}$, Nima Pouladian ${ }^{4}$, Nepton Soltani*5
}

Received: 12 Mar 2017

Published: 6 Feb 2019

\section{Abstract}

Background: Magnesium deficiency plays a key role in obesity and decreases insulin sensitivity. In our previous study, significant evidence was provided for the contribution of oral $\mathrm{Mg}$ supplement that could improve insulin sensitivity and body weight in animal trials. The purpose of the present study was to investigate the effects of an herbal supplement containing $300 \mathrm{mg}$ magnesium sulfate on lipid profile, as well as insulin resistance and secretion in overweight patients.

Methods: Seventy overweight non-diabetic volunteers with Body Mass Index (BMI) $>28 \mathrm{~kg} / \mathrm{m} 2$ were included in a randomized double blind placebo-controlled clinical trial (ethic number HUMS REC.1394.57) and registered in Iranian Registry of Clinical Trials (IRCT2012110124756N2 with registration number 24756). They received either placebo or an herbal supplement capsule containing $300 \mathrm{mg}$ magnesium sulfate $\left(\mathrm{MgSO}_{4}\right)$ for 6 months on a daily basis. Metabolic control, lipid profile and magnesium status were determined at baseline and every three months. Student t-test, repeated measure ANOVA and ANCOVA were used to compare the groups.

Results: There was no significant difference between groups before intervention, but daily Mg supplement for 6 months significantly improved fasting insulin level $(6.71 \pm 0.11$ to $6.27 \pm 0.3$ three months after $\mathrm{Mg}$ therapy, $\mathrm{p}<0.01$ vs. $6.41 \pm 0.11$ in control group $(5.83 \pm 0.063)$ six months after Mg therapy, $\mathrm{p}<0.0001)$, HOMA-IR $(1.52 \pm 0.03$ )in control group to $1.36 \pm 0.03$ after three months Mg therapy, $\mathrm{p}<0.05$ vs $1.37 \pm 0.05$ in control group to $1.22 \pm 0.02$ six months after $\mathrm{Mg}$ therapy, $\mathrm{p}<0.05$ ), high density lipoprotein cholesterol (HDL) (43.57 \pm 0.82 in control group to $43.91 \pm 1.92$ three months after $\mathrm{Mg}$ therapy, $\mathrm{p}<0.001 \mathrm{vs} 43.57 \pm 0.82$ in control group to $46 \pm 0.88$ six months after Mg therapy, $<0.01)$, triglyceride (TG) $(163.17 \pm 6.1$ in control group to $141.2 \pm 5.84$ six months after $g$ therapy, $\mathrm{p}<0.05)$ and low density lipoprotein cholesterol (LDL) (112.62 \pm 3.41 in control group to $104.42 \pm 2.35$ six months after $\mathrm{Mg}$ therapy, $\mathrm{p}<0.05)$.

Conclusion: Oral herbal supplement containing $\mathrm{MgSO}_{4}(300 \mathrm{mg} /$ day) could improve plasma insulin level, lipid profile, and insulin resistance in non-diabetic overweight volunteers.

Keywords: Magnesium, Overweight, Blood glucose, Lipid profile, Insulin resistance

Conflicts of Interest: None declared

Funding: Deputy of Research of Hormozgan University of Medical Sciences

*This work has been published under CC BY-NC-SA 1.0 license.

Copyright $($ Iran University of Medical Sciences

Cite this article as: Solati M, Kazemi L, Shahabi Majd N, Keshavarz M, Pouladian N, Soltani N. Oral herbal supplement containing magnesium sulfate improve metabolic control and insulin resistance in non-diabetic overweight patients: A randomized double blind clinical trial. Med $J$ Islam Repub Iran. 2019 (6 Feb);33:2. https://doi.org/10.47176/mjiri.33.2

\section{Introduction}

Obesity and type 2 diabetes are interrelated. The major foundation for this relationship is the capacity of fatness which stimulates insulin resistance as an essential facet of type 2 diabetes etiology and ties up to an extensive array

Corresponding author: Dr Nepton Soltani, nsoltani@hums.ac.ir

1. Endocrinology and Metabolism Research Center, Hormozgan University of Medical Sciences, Bandar Abbas, Iran

2. Department of Basic Sciences, Faculty of Paramedical Sciences, Shahid Beheshti University of Medical Sciences, Tehran, Iran

3. Department of Physiology, Faculty of Medicine, Tehran University of Medical Sciences, Tehran, Iran

4. English Language Department, school of medicine, Hormozgan University of Medical Sciences, Bandar Abbas, Iran

5. Department of Physiology, School of Medicine, Isfahan University of Medical Sciences, Isfahan, Iran, \& Cardiovascular Research Center for Hormozgan University of Medical Sciences, Bandar Abbas, Iran of other abnormal outcomes including hypertension, hyperlipidemia, atherosclerosis and polycystic ovarian disease (1). Insulin resistance has been shown to impair glucose uptake in type 2 or non-ins ulin dependent diabetes

$\uparrow$ What is "already known” in this topic:

For improve insulin sensitivity and lipid profiles in nondiabetic insulin resistance patients administration of $\mathrm{MgSO}_{4}$ can be useful.

$\rightarrow$ What this article adds:

$\mathrm{Mg}$ shortage has been related with insulin resistance and amplified possibility for type 2 diabetes and fatty liver in adults, so herbal supplement containing $\mathrm{MgSO} 4$ administration can be recommended. 
mellitus (NIDDM) patients (2). The prevalence of overweight between urban population aged above 18 year and under age 18 year is roughly estimated at $21.7 \%$ and $6.1 \%$, respectively (3).

The influence of body fat distribution on insulin sensitivity has been a question of great interest in the literature due to the higher risk of cardiovascular illness and type 2 diabetes in patients, but the exact mediators remain unclear. Magnesium deficiency is said to play a role (4), since it often coincides with insulin resistance in diabetic patients (4). Guerrero-Romero et al showed that a 3-month $\mathrm{MgCl}_{2}$ (2.5 g daily) therapy could enhance beta cells' ability to set off insulin sensitivity in non-diabetic patients with considerable hypomagnesaemia (4). Some researchers (4-6) have provided important data indicating that oral $\mathrm{Mg}$ supplement improves insulin sensitivity even in overweight, non-diabetic and normal magnesium subjects. They emphasize that early optimization of $\mathrm{Mg}$ is necessary to prevent insulin resistance and type 2 diabetes. Kim et al. showed an opposite relationship between magnesium ingestion and diabetes in American new generation. This may be due to the reverse correlation of magnesium intake with systemic inflammation and insulin resistance (7).

In our previous study, we reported that oral magnesium sulfate administration in animal diabetic model could not increase insulin secretion, but could improve insulin sensitivity by an increase in Glucose transporter number 4 (GLIUT4) gene expressions (8). It could also correct blood glucose and lipid profile, and lessen high blood pressure and diabetic vascular complications (9-12). We also found that daily administration of $300 \mathrm{mg}$ magnesium sulfate for 3 months in type 2 diabetic patients, significantly improved fasting blood sugar, 2-hour post prandial glucose, lipid profile, blood pressure, and hepatic enzymes (13). In this study however, due to prevalence of overweight among Iranian population, we used an herbal supplement containing $\mathrm{MgSO}_{4}$ in the non-diabetic obese to measure the efficacy of the drug on insulin resistance.

\section{Methods}

This study was a randomized, double-blind, placebocontrolled trial confirmed by the ethics committee of Hormozgan university of medical sciences (ethic number HUMS REC.1394.5 7) and registered in Iranian Registry of Clinical Trials (IRCT2012110124756N2 with registration number 24756). Data privacy and confidentiality were protected accordingly. All participants and researchers except for two, with randomized participation, were blind to the study protocol. Our colleague who was responsible for sample randomization allocated the participant between groups by using table of random numbers and replacement sample in case if we lost someone. The research flows illustration is presented in Fig. 1.

\section{Participants}

In this study, based on Iranian insulin resistance biomarker and the biostatistician suggestion, the sample size was determined using the following formula:

$$
\mathrm{n}=2 \mathrm{SD}\left(\mathrm{z}_{\alpha / 2}+\mathrm{Z}_{\beta}\right)^{2} / \mathrm{d}^{2} \quad\left(\mathrm{z}_{\alpha / 2}=1.96, \mathrm{Z}_{\beta}=0.84\right)
$$

The inclusion criteria for the overweight volunteers were age (19-69 years), Body Mass Index ( $\left.>28 \mathrm{~kg} / \mathrm{m}^{2}\right)$ (14), adequate cardiovascular and gastrointestinal function and appropriate mobility as well as being non-diabetic, non-pregnant, normotensive and with no dietary restrictions (our patients followed the usual diet, but we asked them to complete the 24 hours food recall form which we prepared for them) or allergies with no history of food allergy or sensitivity to magnesium supplements. Patients who had known kidney diseases (serum creatinin above $1.3 \mathrm{mg} / \mathrm{dl}$ in women and higher than $1.5 \mathrm{mg} / \mathrm{dl}$ in men), raised hepatic enzymes (above 3 folds over normal range), current infections (fewer one month during the study) and chronic inflammatory diseases, cerebrovascular accident (CVA) and acute coronary syndrome (ACS) less than a month during the study, but alcoholics or drug users were omitted. Moreover, patients with insulin, calcium channel blockers and $\mathrm{Mg}$ supplements were also excluded from the study. After an opening screening and face-off

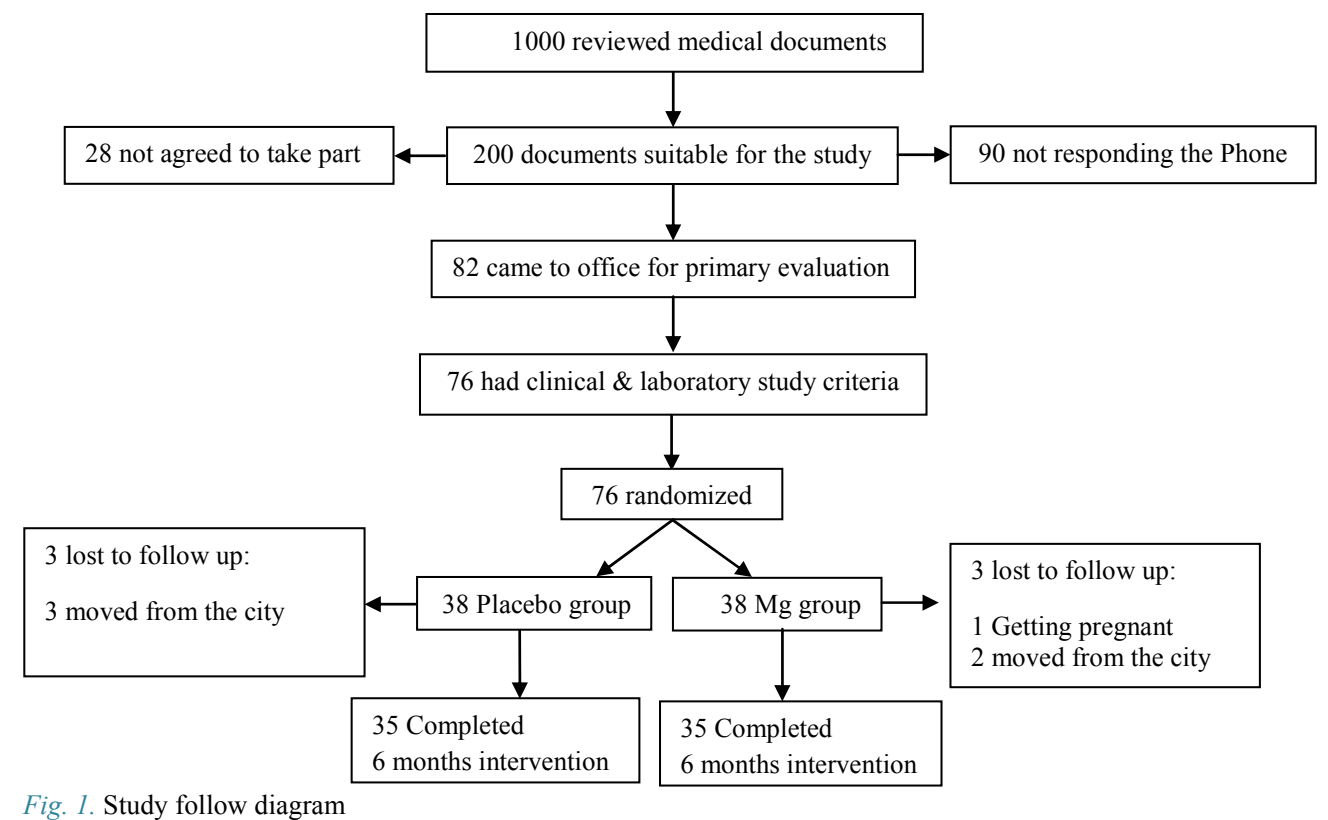


period, participants were randomly chosen and enrolled for the study protocol upon taking informed written consent to get either herbal supplement capsules containing $300 \mathrm{mg} \mathrm{MgSO}_{4}$ or placebo (wheat bran) every morning for 6 months (Fig. 1). Six participants did not complete the whole study while other participants followed the usual diet and physical activity during the course of study.

Participants were visited by an endocrinologist blind to the grouping on a weekly basis. Every 3 months blood sample was taken to measure biomedical markers. Patients and staffs were also unsighted to the group management. The study predictor had access to the data and followed the no-contact rule with the participants during the study.

\section{Study outcomes}

The primary outcome regarding fasting blood glucose concentration and HOMA-IR, measured before intervention and in three-month intervals following the intervention. The secondary outcome related to insulin and lipid profile was also measured in aforementioned times.

\section{Measurements}

Height and weight were measured with light indoor clothing without shoes, using standard protocols. BMI was calculated through weight in kilograms divided by height in square meters (15).

Fasting blood glucose (FBG) was determined every three months using glucose-oxidase method (Glucosemonoreagent kit, Kimia Pajhouhan, Iran). Moreover, enzymatic methods were used to measure total cholesterol (CHOD-PAP cholesterol kit, Pars Azmun, Iran), LDL cholesterol (LDL- C kit, Pars Azmun, Iran), HDL cholesterol (CHOD-PAP cholesterol kit, Pars Azmun, Iran), and triglycerides (GPO-PAP triglycerides kit, Pars Azmun, Iran). Plasma insulin concentrations were determined by ELISA test (Monobind insulin microplate ELISA test, Monobind Inc., USA). Serum Mg levels was measured by colorimetric magnesium kit (Pars Azmun, Iran), and the following formula was used to measure insulin resistance;

HOMA-IR $=$ FBG $(\mathrm{mg} / \mathrm{dl}) \times$ FPI $(\mu \mathrm{u} / \mathrm{ml}) / 405(16)$.

\section{Statistical investigates}

The findings communicated as mean $\pm \mathrm{SD}$, and Kolmogorov-Smirnov test was used to check the normality of all variables.
The possible changes between case and control group before intervention were analyzed by Student's t-test. Comparisons between groups and the possible differences between means of each group during the intervention were conducted with repeated measure of ANOVA and analysis of covariance (ANCOVA). For the analyses, SPSS software, version 13 , was used and $\mathrm{P}<0.05$ accepted as statistically important.

\section{Results}

Seventy six participants were included in this study, and unsystematically allocated to Mg-treated $(n=38)$ and placebo $(\mathrm{n}=38)$ groups. In the $\mathrm{Mg}$ group, participants took $300 \mathrm{mg}$ magnesium sulfate in form of herbal supplement capsules on a daily basis whereas in the placebo group, participants received placebo capsules. Three participants in each group were excluded during the follow-up period due to migration or failure to meet eligibility criteria. In all, thirty-five participants in $\mathrm{Mg}$ group and thirty-five in placebo group successfully completed the six-month intervention (Fig. 1). Study variables before and after the interventions are shown in Tables 1 and 2.

No statistical differences were seen between the two groups before intervention (Table 1). However, administration of herbal supplement containing $300 \mathrm{mg}$ magnesium sulfate for 3 months significantly decreases plasma insulin level and HOMA-IR. Our results also showed that plasma HDL level significantly improved during the course of 3 months following $\mathrm{Mg}$ supplementation (Table 2).

The six-month $\mathrm{Mg}$ supplementation significantly increased plasma $\mathrm{Mg}$ level compared to placebo group, while plasma TG and LDL levels significantly decreased in the Mg-treated group (Table 2).

Our results also showed a significant decrease in plasma insulin level and HOMA-IR in the Mg-preserved group compared to the control group after six months of magnesium sulfate supplementation (Table 2). In Mg-treated group plasma HDL level and the HDL/TG ratio also significantly increased compared to the placebo group (Table 2).

\section{Discussion}

In this randomized double blind clinical trial in overweight participants, herbal supplement containing $300 \mathrm{mg}$

Table 1. Study variables in $\mathrm{Mg}$ treated and placebo group before intervention (data are expressed as mean $\pm \mathrm{SD}$ and comparisons between groups were analyzed using the Student's t-test)

\begin{tabular}{lccc}
\hline Variable & Placebo group (35) & Mg-treated group (35) & p \\
\hline Sex (F/M) & $15 / 20$ & $16 / 19$ & - \\
Age & $40.65 \pm 12.74$ & $40.73 \pm 11.9$ & 0.64 \\
BMI (kg/m2) & $28.92 \pm 0.19$ & $28.81 \pm 0.15$ & 0.34 \\
TG (mg/dl) & $165.37 \pm 7.46$ & $163.22 \pm 7.67$ & 0.63 \\
Cholesterol (mg/dl) & $140.02 \pm 4.59$ & $143.2 \pm 5.8$ & 0.21 \\
LDL 9mg/dl) & $115.48 \pm 3.94$ & $117.11 \pm 3.01$ & 0.22 \\
HDL (mg/dl) & $44.4 \pm 0.81$ & $43.28 \pm 1.09$ & 0.28 \\
HDL/TG & $0.28 \pm 0.01$ & $0.28 \pm 0.01$ & 0.39 \\
Insulin (qIU/ml) & $6.67 \pm 0.76$ & $6.66 \pm 0.08$ & 0.98 \\
HOMA-IR & $1.5 \pm 0.03$ & $1.49 \pm 0.02$ & 0.39 \\
FBS (mg/dl) & $91.54 \pm 1.5$ & $90.68 \pm 1.43$ & 0.55 \\
Mg (mg/dl) & $1.93 \pm 0.02$ & $1.98 \pm 0.03$ & 0.54 \\
\hline Abbreviation, Body mass index (BMI), Fasting blood glucose (FBG), Low density lipoprotein cholesterol (LDL), High density lipoprotein cholesterol (HDL),
\end{tabular}

triglyceride $(\mathrm{TG})$, magnesium $(\mathrm{Mg})$, and HOMA-IR $=$ FBG $(\mathrm{mg} / \mathrm{dl}) \times \mathrm{FPI}(\mu \mathrm{u} / \mathrm{ml}) / 405)$. 
Table 2. Study variables in Mg treated and placebo groups 3 months (M3) and 6 months (M6) after intervention and comparisons between groups were analyzed using the repeated measure and ANCOVA (data are expressed as mean \pm SEM)

\begin{tabular}{|c|c|c|c|c|c|c|}
\hline & \multicolumn{3}{|c|}{ Placebo group } & \multicolumn{2}{|c|}{ Mg-treated group } & \multirow[b]{2}{*}{ M6 } \\
\hline & M0 & $\mathrm{M} 3$ & M6 & M0 & M3 & \\
\hline $\operatorname{Sex}(\mathrm{F} / \mathrm{M})$ & $15 / 20$ & $15 / 20$ & $15 / 20$ & $16 / 19$ & $16 / 19$ & $16 / 19$ \\
\hline BMI & $28.92 \pm 0.19$ & $28.92 \pm 0.19$ & $28.92 \pm 0.19$ & $28.81 \pm 0.15$ & $28.81 \pm 0.15$ & $28.81 \pm 0.15$ \\
\hline TG & $165.37 \pm 7.46$ & $159.21 \pm 6.92$ & $163.17 \pm 6.1$ & $163.22 \pm 7.67$ & $159.11 \pm 6.67$ & $141.2 \pm 5.84^{\$}$ \\
\hline Cholesterol & $140.02 \pm 4.59$ & $142.82 \pm 4.3$ & $139.45 \pm 5.28$ & $143.2 \pm 5.8$ & $137.65 \pm 5.1$ & $131.34 \pm 4.27$ \\
\hline LDL & $115.48 \pm 3.94$ & $116.48 \pm 3.69$ & $112.62 \pm 3.41$ & $117.11 \pm 3.01$ & $110.62 \pm 3.08$ & $104.42 \pm 2.35^{\S}$ \\
\hline HDL & $44.4 \pm 0.81$ & $44.34 \pm 0.91$ & $43.57 \pm 0.82$ & $43.28 \pm 1.09$ & $43.91 \pm 1.92^{*}$ & $46 \pm 0.88^{\#}$ \\
\hline $\mathrm{HDL} / \mathrm{TG}$ & $0.28 \pm 0.01$ & $0.29 \pm 0.01$ & $0.27 \pm 0.01$ & $0.28 \pm 0.01$ & $0.29 \pm 0.01$ & $0.34 \pm 0.01^{*}$ \\
\hline Insulin & $6.67 \pm 0.76$ & $6.71 \pm 0.11$ & $6.41 \pm 0.11$ & $6.66 \pm 0.08$ & $6.27 \pm 0.1^{\#}$ & $5.83 \pm 0.06^{*}$ \\
\hline HOMA-IR & $1.5 \pm 0.03$ & $1.52 \pm 0.03$ & $1.37 \pm 0.05$ & $1.49 \pm 0.02$ & $1.36 \pm 0.03^{\S}$ & $1.22 \pm 0.02^{\$}$ \\
\hline FBS & $91.54 \pm 1.5$ & $91.85 \pm 1.58$ & $85.53 \pm 3.1$ & $90.68 \pm 1.43$ & $88.34 \pm 1.51$ & $84.85 \pm 1.48$ \\
\hline $\mathrm{Mg}$ & $1.93 \pm 0.02$ & $1.97 \pm 0.02$ & $1.93 \pm 0.02$ & $1.98 \pm 0.03$ & $2.02 \pm 0.02$ & $2.12 \pm 0.02^{*}$ \\
\hline $\begin{array}{l}\text { Abbreviation: } \\
\text { triglyceride (T } \\
\text { * Mg-treated-1 } \\
\text { \# Mg-treated-1 } \\
\text { \$ Mg-treated-1 }\end{array}$ & $\begin{array}{l}\text { Placebo-M3 an } \\
\text { Placebo-M3 an } \\
\text { Placebo-M3 an }\end{array}$ & $\begin{array}{l}\text { ated-M6 vs Plac } \\
\text { ated-M6 vs Plac } \\
\text { ated-M6 vs Plac }\end{array}$ & $\begin{array}{l}\text { Low density } \\
<\text { FPI }(\mu \mathrm{u} / \mathrm{ml}) \\
(\mathrm{p}<0.0001) \\
\mathrm{p}<0.001) \\
\mathrm{p}<0.05)\end{array}$ & & & \\
\hline
\end{tabular}

magnesium sulfate for six months significantly decreased plasma TG, LDL, Insulin levels and HOMA-IR, whereas a significant increase in plasma $\mathrm{Mg}$ level and HDL was witnessed.

It has been previously documented that oral $\mathrm{MgSO}_{4}$ administration in streptozotocin (STZ) diabetic rat could decrease plasma glucose level via increasing GLUT4 gene expression in the muscles without any changes in insulin secretion (8). It seems that $\mathrm{Mg}$ can decrease insulin resistance and improve lipid profiles, thus based on our previous study in type 2 diabetic patients (13) and STZdiabetic rats (8-12), this study was planned to determine whether oral herbal supplement containing $\mathrm{MgSO}_{4}$ improves lipid profile, insulin resistance and insulin secretion in over-weight non diabetic patients.

The results of the present study showed that plasma magnesium level increased six months after $\mathrm{MgSO}_{4}$ therapy. Some controversy exists in the data in regard to the role of magnesium in insulin secretion. Few studies showed that, low serum magnesium concentration plays a role in hyperinsulinemia in non-diabetic individuals (4). Although it is believed that magnesium plays a role as a second messenger for insulin pathway and $\mathrm{Mg}^{2+}$ administration can decrease blood sugar after diabetes induction via insulin secretion and action, but the relationship between plasma magnesium level and glucose metabolism is independent of insulin secretion in diabetic patient (4). In our previous study, it was observed that $\mathrm{MgSO}_{4}$ administration in type 2 diabetic patients for 3 months could not increase insulin secretion (13). It was also shown that oral $\mathrm{MgSO}_{4}$ administration in STZ-diabetic rats for eight weeks did not increase insulin secretion (8) and glucosestimulated insulin secretion is concentration-dependent in nature. However, in the presence of magnesium, insulin secretion did not show any changes (8). Our previous study supported the hypothesis that magnesium could decrease blood glucose via increase in GLUT4 mRNA expression, but not by increasing insulin secretion (8). GLUT4 is the main glucose carrier isoform in the insulin target tissues like adipose and skeletal muscle tissues and thus it is a very good index for insulin sensitivity (8). Our findings in the present study indicated that plasma insulin level and HOMA-IR (the index for insulin resistance) de- creased after six months of Mg-therapy. This finding confirms our previous studies (8) and other studies as well (5, $6,17-20)$. This data supports the idea that magnesium may play an important role in insulin sensitivity.

A meta-analysis of 9 randomized trials in diabetic patients reported that magnesium therapy of 4-16 weeks was effective in increasing HDL level but it had no effect on total cholesterol, LDL and triglyceride concentrations (21). Clinical data regarding the effect of $\mathrm{Mg}$ supplementation on serum lipids in diabetic patients conflict, are positive as several studies $(5,6,17-20)$, such as Hadjistavri et al showed that oral magnesium supplementation 600 $\mathrm{mg}$ /day for 12 weeks in diabetic patients could decreases serum total cholesterol, LDL and triglyceride but it increases HDL level at the same time (13). On the other hand, other studies have reported zero or negative effects (22). Our previous studies in STZ-diabetic animal model (8-12) and type 2 diabetic patients (13) showed that oral $\mathrm{MgSO}_{4}$ administration had a beneficial effect on some lipid profiles.

In the present study, it was shown that $\mathrm{Mg}$ supplementation associated with significant reductions in serum TG and LDL levels, parallel with a significant increase in HDL levels and HDL/TG ratio, whereas in the control group no effect on lipid profile was evident. These findings can be enlightened via the role of $\mathrm{Mg}$ in the lipoprotein lipase enzyme action (13). Mg deficiency improves catecholamine excretion which augmented lipolysis. Enhancement of lipolysis and subsequent elevation of plasma free fatty acids may lead to an increase in VLDL and TG synthesis and secretion and elevated plasma TG concentration (13).

The participants of the present study were volunteers and we also could not use euglycemic insulin clamp technique, a standard method for insulin secretion and resistance measurement, because it was very expensive and invasive for our patients. This was a limitation and weak point of this study.

\section{Conclusion}

From the results of the present study it can be concluded that herbal supplement containing $\mathrm{MgSO}_{4}$ improves insulin sensitivity and has a beneficial effect on lipid-lowering 
and it could be of great help for non-diabetic insulin resistance patients. The $\mathrm{Mg}$ deficiency plays an important role in insulin resistance and increased risk for type 2 diabetes and fatty liver in adults; hence $\mathrm{Mg}$ therapy is suggested as a low-cost, easy to use and natural component for prevention of type 2 diabetes.

\section{Acknowledgments}

This study was supported by Deputy of Research of Hormozgan University of Medical Sciences. We appreciate Niak Pharmaceuticals Co for providing sincere medicine (Gorgan, I.R.Iran). All rights of the drug formulation reserved to Dr. Naghi Shahabi majd.

\section{Conflict of Interests}

The authors declare that they have no competing interests.

\section{References}

1. Kangduk C, Young-Bum K. Molecular Mechanism of Insulin Resistance in Obesity and Type 2 Diabetes. Korean J Intern Med. 2010;25(2):119-129.

2. Reddy J, Singh M, Bangit R, Batsell R. The role of insulin resistance in the pathogenesis of atherosclerotic cardiovascular disease: an updated review J Cardiovasc Med. 2010;11(9):633-647.

3. Rahmani A, Sayehmiri K, Asadollahi K, Sarokhani D, Islami F, Sarokhani M. Investigation of the Prevalence of Obesity in Iran: a Systematic Review and Meta-Analysis Study. Acta Med Iran. 2015;53(10):596-607.

4. Guerrero-Romero F, Rodríguez-Morán M. Magnesium improves the beta-cell function to compensate variation of insulin sensitivity: double-blind, randomized clinical trial. Eur $\mathrm{J}$ Clin Invest. 2011;41(4):405-10.

5. Kim DJ, Xun P, Liu K, Loria C, Yokota K, Jacobs DR, He K. Magnesium Intake in Relation to Systemic Inflammation, Insulin Resistance, and the Incidence of Diabetes. Diabetes Care. 2010;33(12): 2604-2610.

6. Mooren F, Krüger K, Völker K, Golf S, Wadepuhl M, Kraus A. Oral magnesium supplementation reduces insulin resistance in nondiabetic subjects-a double-blind, placebo-controlled, randomized trial. Diabetes Obes Metab. 2011;13(3):281-4.

7. Kim DJ, Xun P, Liu K, Loria C, Yokota K, Jacobs DR, et al. Magnesium intake in relation to systemic inflammation, insulin resistance, and the incidence of diabetes. Diabetes Care. 2010;33(12):2604-10.

8. Solaimani H, Soltani N, MaleKzadeh K, Sohrabipour S, Zhang N, Nasri S, et al. Modulation of GLUT-4 expression by oral administration of $\mathrm{Mg} 2+$ to control sugar level in STZ-induced diabetic rat. Can J Physiol Pharmacol. 2014;92(6).

9. Soltani N, Keshavarz M, Dehpour AR. Effect of oral magnesium sulfate administration on blood pressure and lipid profile in streptozocin diabetic rat. Eur J Pharmacol. 2007;560(2):201-5.

10. Soltani N, Keshavarz M, Sohanaki H, Dehpour AR, Asl SZ. Oral magnesium administration prevents vascular complications in STZdiabetic rats. Life Sci. 2005;76(13):1455-64

11. Soltani N, Keshavarz M, Minaii B, Mirershadi F, Asl SZ, Dehpour AR. Effects of administration of oral magnesium on plasma glucose and pathological changes in the aorta and pancreas of diabetic rats. Clin Exp Pharmacol Physiol. 2005;32(8):604-10.

12. Soltani N, Keshavarz M, Sohanaki H, Zahedi Asl S, Reza Dehpour A. Relaxatory effect of magnesium on mesenteric vascular beds differs from normal and streptozotocin induced diabetic rats. Eur J Pharmacol. 2005;508(1):177-81.

13. Solati M, Ouspid E Hosseini S, Soltani N, Keshavarz M, Dehghani M. Oral magnesium supplementation in type II diabetic patients. Med J Islam Repub Iran. 2014;28:1-8.

14. Ayatollahi SM, Ghoreshizadeh Z. Prevalence of obesity and overweight among adults in Iran. Obes Rev. 2010;11(5):335-7.

15. Panel NOEIE. Clinical guidelines on the identification, evaluation, and treatment of overweight and obesity in adults. 1998.

16. Kurtolu S, Hatipolu N, Mazcolu M, Kendirci M, Keskin M, Kondolot M. Insulin Resistance in Obese Children and Adolescents: HOMA-IR Cut-Off Levels in the Prepubertal and Pubertal Periods. J Clin Res Ped Endo. 2010;2(3):100-106.

17. Mooren FC, Krüger K, Völker k, Golf SW, Wadepuhl M, Kraus A. Oral magnesium supplementation reduces insulin resistance in nondiabetic subjects - a double-blind, placebo-controlled, randomized triąl. J Clin Res Ped Endo. 2011;13(3):281-284.

18. Hadjistavri LS, Sarafidis PA, Georgianos PI, Tziolas IM, Aroditis $\mathrm{CP}$, Hitoglou-Makedou A, et al. Beneficial effects of oral magnesium supplementation on insulin sensitivity and serum lipid profile. Med Sci Monit. 2010;16(6)

19. Chaudhary P, Sharma R, Bansal DD. Implications of Magnesium Deficiency in Type 2 Diabetes: A Review. Biol Trace Elem Res. 2010;134(2):119-129.

20. Corre T, Arjona FJ, Hayward C, Youhanna S, de Baaij JHF, Belge $\mathrm{H}$, et al. Genome-Wide Meta-Analysis Unravels Interactions between Magnesium Homeostasis and Metabolic Phenotypes. J Am Soc Nephrol. 2018;29(1):335-348.

21. Wolide AD, Zawdie B, Alemayehu T, Tadesse S. Association of trace metal elements with lipid profiles in type 2 diabetes mellitus patients: a cross sectional study. BMC Endocr Disord. 2017;17(1):64.

22. Hadjistavri LS, Sarafidis PA, Georgianos PI, Tziolas IM, Aroditis $\mathrm{CP}$, Hitoglou-Makedou A, et al. Beneficial effects of oral magnesium supplementation on insulin sensitivity and serum lipid profile. Med Sci Monit. 2010;16(6):307-12. 